\title{
Review of: "In situ Raman spectroscopy reveals the structure and dissociation of interfacial water"
}

\author{
Changqing Sun ${ }^{1}$ \\ 1 Nanyang Technological University
}

Potential competing interests: The author(s) declared that no potential competing interests exist.

A nice piece of work done using Raman spectroscopy to examine the joint effect of molecular undercoordination, ionic polarization, and directional electric field on the Raman frequency shift and catalyticity. Discoveries of the blueshift of $\mathrm{H}-\mathrm{O}$ phonon frequency and the $\mathrm{O}: \mathrm{H}-\mathrm{O}$ bending mode redshift evidence the $\mathrm{O}: \mathrm{H}-\mathrm{O}$ bond cooperativity and polarizability (HBCP). Water follows the respective rules in responding to the perturbations, which is very encouraging for deep understanding and engineering of liquid water. For more fundamental rules, please refer to:

1. Sun, C. Q., et al. (2013). "Density, Elasticity, and Stability Anomalies of Water Molecules with Fewer than Four Neighbors." JPCL 4: 2565-2570.

2. Sun, C. Q. (2020). "Water electrification: Principles and applications." Adv Colloid Interf Sci 282: 102188.

3. Sun, C. Q., et al. (2019). "Hydration of Hofmeister ions." Adv Colloid Interf 268: 1-24.

4. Huang, Y. L., et al. (2015). "Hydrogen-bond relaxation dynamics: Resolving mysteries of water ice." Coord Chem Rev 285: 109-165. 